\title{
Hombres con diabetes mellitus tipo 2: autoeficacia y factores psicológicos que influyen en el autocuidado
}

\section{Type 2 diabetes mellitus among men: psychological factors influencing self-care efficacy}

\section{Homens com diabetes mellitus tipo 2: autoeficácia e fatores psicológicos que influem no autocuidado}

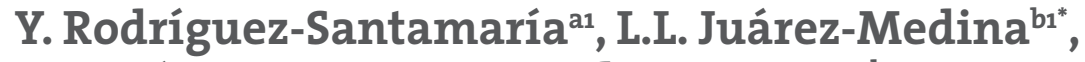 \\ M.L. Zúñiga-Vargas ${ }^{\mathrm{c}}$, F. Cadena-Santos ${ }^{\mathrm{d} 1}$, \\ G. Mendoza-Catalán ${ }^{\mathrm{e} 2}$ \\ ORCID: \\ $\begin{array}{ll}{ }^{a} \text { https://orcid.org/oooo-0002-7010-2753 } & { }^{b} \text { https://orcid.org/oooo-ooo2-9656-4198 } \\ { }^{c} \text { https://orcid.org/o0oo-002-4028-9190 } & { }^{d} \text { https://orcid.org/oooo-ooo1-8792-692X } \\ \text { e https://orcid.org/oooo-0oo2-5061-2457 } & \end{array}$
}

${ }^{1}$ Universidad Autónoma de Tamaulipas, Facultad de Enfermería, Nuevo Laredo, Tamaulipas, México

${ }^{2}$ Universidad Intercultural del Estado de Puebla, Licenciatura de Enfermería, División de Ciencias de la Salud, Huehuetla, Puebla, México

Recibido: 15 julio 2019 / Aceptado: 12 diciembre 2019

RESUMEN

Introducción: La prevalencia global de diabetes mellitus tipo 2 (DMT2) en los hombres es alta. Para el tratamiento de la enfermedad el hombre debe realizar acciones de autocuidado, por lo que es importante conocer factores relacionados con su cumplimiento.

Objetivo: Identificar la relación entre variables demográficas, clínicas, la angustia, la depresión, la autoeficacia y su influencia sobre el autocuidado en hombres con DMT2.

Métodos: Estudio transversal y correlacional, en una muestra de 96 hombres con DMT2, usuarios de 13 centros de salud. Los instrumentos utilizados fueron el cuestionario de acciones de cuidado en diabetes, la escala Self-Efficacy for Diabetes, la escala depresión (CES-D) y la escala angustia por diabetes (DDS). 
Resultados: El autocuidado se correlacionó de manera positiva con la autoeficacia y negativamente con la angustia por diabetes. Las variables que explicaron el autocuidado con el $47.7 \%$ de varianza fueron: la autoeficacia $(\beta=.39)$, el consumo de alcohol $(\beta=-.29)$, las horas sentado/parado $(\beta=-.27)$, el tiempo de diagnóstico $(\beta=-.22)$ y la edad $(\beta=.18)$.

Discusión: Los hombres tuvieron un bajo autocuidado y mantienen conductas de riesgo, lo cual puede atribuirse a sus creencias, estilo de vida o al tipo de trabajo que desempeñan.

Conclusiones: Los resultados ofrecen evidencia sobre factores que influyen en el autocuidado de hombres con DMT2, que pueden orientar a los profesionales de enfermería en la atención del paciente. Se sugiere realizar investigaciones de enfermería en hombres, que contribuyan a mejorar la autoeficacia y el autocuidado.

Palabras clave: Autocuidado; autoeficacia; hombres; diabetes mellitus tipo 2; México.

\section{ABSTRACT}

Introduction: The global prevalence of type 2 diabetes mellitus among men is high; and these patients need to follow self-care routines. Therefore, knowing the factors which can affect their level of compliance is an important issue for nursing.

Objective: To identify the relationship between demographic and clinical variables such as anguish and depression, and self-care efficacy in men suffering from type 2 diabetes mellitus.

Methods: This a transversal and correlational study on a sample of 96 men with type 2 diabetes mellitus who were registered in 13 health centers. The instruments used were the Questionnaire of Actions of Care in Diabetes, the Self-Efficacy for Diabetes Scale (SED), the Mexico Center of Epidemiological Studies of Depression Scale (CES-D), and the Diabetes Distress Scale (DDS).

Results: Self-care was positively correlated to self-efficacy, and negatively correlated to diabetes-related anguish and depression. Variables which accounted for $47.7 \%$ of the self-care variance were: self-efficacy ( $\beta=.39$ ), alcohol consumption $(\beta=-.29)$, number of hours sitting or standing ( $\beta=-.27)$, years of being diagnosed $(\beta=-.22)$, and age $(\beta=.18)$.

Discussion: The sample demonstrated a low level of diabetes self-care which included risky behaviors. This attitude could be a result of the patients' beliefs, lifestyles, and/or type of job. Conclusions: The findings provide evidence on the factors which can influence self-care among men with type 2 diabetes. These results can orient nursing professionals while providing patient attention. Further related studies are suggested in order to help improve efficacy in type 2 diabetes self-care.

Keywords: Self-care; self-efficacy; men; type 2 diabetes mellitus; México.

\section{RESUMO}

Introdução: A prevalência global de diabetes mellitus tipo 2 (DMT2) nos homens é alta. Para o tratamento da doença o homem deve realizar ações de autocuidado, pelo que é importante conhecer fatores relacionados com sua conformidade.

Objetivo: Identificar a relação entre variáveis demográficas, clínicas, a angustia, a depressão, a autoeficácia e sua influência sobre o autocuidado em homens com DMT2.

Métodos: Estudo transversal e correlacional, em uma amostra de 96 homens com DMT2, usuários de 13 centros de saúde. Os instrumentos utilizados foram o questionário de ações de cuidado em diabetes, a escala Self-Efficacy for Diabetes, a escala depressão (CES-D) e a escala angustia por diabetes (DDS). 
Resultados: O autocuidado correlacionou-se de maneira positiva com a autoeficácia e negativamente com a angustia por diabetes. As variáveis que explicaram o autocuidado com o 47.7\% de variância foram: a autoeficácia ( $\beta=.39$ ), o consumo de álcool ( $\beta=-.29)$, as horas sentado/parado $(\beta=-.27)$, o tempo de diagnóstico $(\beta=-.22)$ e a idade $(\beta=.18)$.

Discussão: Os homens tiveram um baixo autocuidado e mantém condutas de risco, o qual pode atribuir-se a suas crenças, estilo de vida ou ao tipo de trabalho que desempenham.

Conclusões: Os resultados oferecem evidência sobre fatores que influem no autocuidado de homens com DMT2, que podem orientar aos profissionais de enfermagem na atenção do paciente. Sugere-se realizar pesquisas de enfermagem em homens, que contribuam a melhorar a autoeficácia e o autocuidado.

Palavras chave: Autocuidado; autoeficácia; homens; diabetes mellitus tipo 2; México.

\section{INTRODUCCIÓN}

La Diabetes Mellitus Tipo 2 (DMT2) se considera un problema de salud pública, a nivel mundial, debido a las altas tasas de mortalidad y prevalencia en adultos (8.8\%); se prevé que para el año 2045 sea la séptima causa de muerte'. En México, la enfermedad muestra tendencia al incremento, ya que en 2012 la tasa fue de $9.2 \%$ y en 2016 de $9.4 \%^{2}$. Además, la DMT2 es la segunda causa de muerte después de las enfermedades isquémicas del corazón3. En cuanto al género, de manera global la prevalencia es mayor en hombres (9.1\%) que en mujeres (8.4\%). El tratamiento de la DMT2 es complejo; los enfermos deben cumplir con acciones de autocuidado como el apego farmacológico, la alimentación saludable, el ejercicio, el no consumo de alcohol o tabaco y el manejo adecuado de los aspectos psicológicos4-6.

El autocuidado de la DMT2 es considerado como una conducta que mejora el control glucémico ${ }^{7-9}$ y ayuda a retrasar las complicaciones de la misma ${ }^{10}$. El autocuidado en los hombres también se ha estudiado en relación con factores sociodemográficos como el nivel educativo, la edad, la escolaridad y la ocupación $\mathrm{n}^{11}$. Sin embargo, el género es un factor que puede condicionar a las personas para efectuar acciones de salud ${ }^{12}$. Se ha reportado que los hombres con DMT2 tienen menor apego al estilo de vida saludable $\mathrm{e}^{12-15}$. Lo anterior se puede explicar en parte por la construcción del modelo hegemónico de masculinidad, el cual considera que el hombre posee características como independencia, agresividad, competencia, fuerza y dominio, las cuales pueden predisponer a presentar conductas de riesgo $^{12}$, así como al control de emociones y a evitar buscar ayuda. Todo lo mencionado se ve reflejado en el contexto cultural mexicano y se relaciona con algunos factores que influyen para que los hombres no cumplan con su autocuidado, tales como la falta de tiempo, el trabajo, la falta de percepción de la gravedad de la enfermedad y sus complicaciones ${ }^{16}$.

No obstante, la autoeficacia es considerada como el factor que mayor predice el autocuidado. El constructo de autoeficacia fue introducido por Bandura ${ }^{17}$, en su teoría social cognitiva; se refiere a la capacidad que tienen las personas de percibir su competencia y eficacia para desempeñarse en una situación determinada. La autoeficacia en la diabetes es el conjunto de habilidades y conocimientos que desarrolla el paciente para poder realizar de manera efectiva su autocuidado. Está asociada con mayor nivel educativo y con haber recibido educación en diabetes ${ }^{18}$; asimismo, favorece el control glucémico, el autocuidado ${ }^{7-9,18-24}$ y la calidad de vida ${ }^{25}$. Sin embargo, la autoeficacia se ve afectada por aspectos psicológicos como la depresión y la angustia7, 20, 21.

En pacientes con DMT2, las mujeres presentan mayores problemas psicológicos como angustia y depresión. ${ }^{15,26}$ Esta última es más frecuente en personas solteras, sin trabajo y que presenten 
complicaciones ${ }^{27,28}$. Sin embargo, se ha reportado que los hombres que presentan síntomas depresivos abandonan su tratamiento $0^{8,16}$ y presentan menor autoeficacia ${ }^{19}$. Generalmente, no logran identificar los síntomas depresivos; algunos llegan a confundirlos con estrés o cansancio. En ellos mostrar síntomas depresivos puede causar estigma social o vergüenza ${ }^{29}$; pueden ocultar sus emociones y negar cualquier problema mental, resaltando su imagen masculina mediante conductas estoicas ${ }^{11}$. La depresión y la angustia por diabetes en muchas ocasiones no se diagnostican o se consideran iguales, debido a la semejanza de los síntomas; por lo tanto, es importante distinguirlos para brindar al paciente el tratamiento adecuado ${ }^{26,30}$.

La angustia hace referencia a la situación que vive un paciente con DMT2 cuando se siente frustrado, enojado o desanimado por tener la enfermedad y lo que implica el tratamiento ${ }^{31}$. En esta situación, los hombres presentan mayor angustia por el régimen terapéutico ${ }^{32} \mathrm{y}$ con mayor tiempo de evolución; mientras que en las personas que realizan actividad física y tienen un plan de alimentación, ésta tiende a disminuir ${ }^{33}$. Las personas con altos niveles de angustia por diabetes cumplen menos con su autocuidado ${ }^{34,35}$, tienen menor calidad de vida relacionada a la salud ${ }^{36}$, presentan mayores síntomas depresivos ${ }^{26,28}$, baja adherencia al tratamiento farmacológico y la falta de ejercicio ${ }^{35}$. También, puede provocar inseguridad en el manejo y sensación de frustración, lo cual puede afectar en la autoeficacia.

La caracterización clínico-epidemiológica de la población es necesaria para identificar posibles barreras en el manejo de la enfermedad, debido a que los valores clínicos como el nivel de glucosa, presión arterial y el peso están relacionados con el automanejo y autocuidado del paciente ${ }^{6}$.

Al considerar la importancia de los factores antes mencionados sobre el autocuidado de los hombres con DMT2, se hace necesario investigar la población de Nuevo Laredo, Tamaulipas. Primero, por no existir el antecedente de estudios que reúnan estas temáticas y, segundo, con el propósito de mejorar las prácticas de autocuidado de los pacientes con base en los resultados obtenidos. Por lo expuesto anteriormente, el objetivo del presente estudio es identificar la relación que existe entre variables sociodemográficas, clínicas, la angustia, la depresión, la autoeficacia y su influencia sobre el autocuidado en hombres con DMT2.

\section{MATERIAL Y MÉTODOS}

Diseño transversal y correlacional. La muestra fue no probabilística, conformada por 96 hombres con DMT2. El reclutamiento se realizó en 13 centros de salud del Municipio de Nuevo Laredo, Tamaulipas, correspondientes a la Jurisdicción Sanitaria No. V de la Secretaría de Salud. A través del censo, durante los meses de marzo y abril de 2018, se identificaron a los participantes; estos fueron varones que acudieron a la consulta para el control de su diabetes.

Los criterios de inclusión fueron: varones, entre 18-59 años, con al menos un año de evolución de la DMT2 y que aceptaron y firmaron el consentimiento informado para el estudio. Se excluyeron a los hombres con algún padecimiento médico que causara alguna limitación para la movilidad, así como quienes no sabían leer ni escribir.

La recolección de los datos se realizó previa a la consulta médica en un área asignada por cada unidad de salud. En primera instancia, se realizaron las mediciones de peso y talla para calcular el indicador antropométrico del estado nutricional (IMC), dividiendo los kilogramos entre su talla en metros cuadrados $\left(\mathrm{kg} / \mathrm{m}^{2}\right)$ como se establece en la NOM-008-SSA3-201737, y la circunferencia de cintura de acuerdo con lo que indica la PROY-NOM-O15-SSA2-201838, así como la presión arterial siguiendo el procedimiento estandarizado39. Enseguida, se evaluó la glicemia preprandial y postprandial, según 
las condiciones del paciente para determinar el control glucémico ${ }^{38}$, medida por punción capilar utilizando un glucómetro de la marca Accu-Check Performa, el cual es un medidor con precisión de acuerdo con las normas ISO15197:2013.

Además, se incluyó una cédula de datos personales con información demográfica (edad de los participantes, escolaridad, estado civil y ocupación), clínica (presión arterial, IMC, cintura, glicemia preprandial y postprandial, años con diabetes, tipo de tratamiento, comorbilidades, monitoreo de la glucosa, si alguna vez en la vida ha recibido educación en diabetes y si actualmente tiene problemas sexuales), así como el tiempo que permanece sentado/parado, consumo de alcohol y tabaco; también, cuatro instrumentos previamente validados.

Cuestionario de acciones de cuidado en diabetes ${ }^{40}$ consta de 11 ítems para medir las actividades que la persona realiza para controlar su diabetes; las opciones de respuesta se establecen en tres tipos de patrones: a) escala del 1 (siempre) al 5 (nunca) para las preguntas 1, 10 y 11; b) escala del 1 (o\%) al 5 (100\%) en las preguntas 2, 3, 4, 5, 7 y 9 y, una escala que va de o al 7 (preguntas 6 y 8) que corresponden al número de días en los que la persona realizó actividades durante la semana. Los ítems 1, 4, 5, 10 y 11 se evalúan a la inversa; la puntuación original osciló entre 9 y 59, el resultado se transformó en valores de o a 100, donde a mayor puntuación mejor fue el autocuidado; con un alfa de Cronbach de $0.66^{41}$ que en este estudio fue de .73 .

Escala de autoeficacia para la diabetes (Self-Efficacy for Diabetes) ${ }^{42}$, con ocho items, para evaluar la seguridad de la persona para realizar actividades correspondientes al cuidado de su enfermedad. Las opciones de respuesta fueron de 1 (nada seguro) al 10 (totalmente seguro); el puntaje final osciló entre 8 y 80 ; el resultado se transformó en valores de o a 100; mayor puntaje indicó mejor autoeficacia, con alfa de Cronbach de $0.85^{42}$, similar en esta muestra con 87 .

Escala de depresión (Center for Epidemiological Studies-Depression [CES-D])43, con 20 reactivos, para valorar cuántos días a la semana se han tenido síntomas depresivos. Las respuestas fueron de o (ningún día) al 3 (todos los días) y los ítems 4, 8, 12 y 16 se evalúan a la inversa; el puntaje original osciló entre o y 6o; puntuación elevada indica mayor gravedad de los síntomas depresivos. El punto de corte sugerido en población mexicana ${ }^{44}$ fue 35 puntos, lo que permitió identificar posible depresión en adultos; con un alfa de Cronbach reportada de $0.84^{45} \mathrm{y}$ de .91 en este estudio.

Escala de distress o angustia por la diabetes (The Diabetes Distress Scale [DDS]) ${ }^{31}$, con 17 ítems, para evaluar diversas situaciones que pueden generar angustia en personas con diabetes. Las opciones de respuesta fueron de 1 (no es problema) al 6 (es un problema muy grave); el puntaje final se obtiene realizando la sumatoria de las respuestas y dividiendo el total entre 17, un resultado mayor o igual a 3 puntos indica angustia moderada, lo que requiere atención clínica; esta escala tuvo un alfa de Cronbach de $0.93^{31}$, similar a este estudio con .95 .

El estudio fue aprobado por el Comité de Investigación y Ética de la Facultad de Enfermería-UAT con registro CA-SC1O; se apegó a lo dispuesto en la Ley General de Salud en Materia de Investigación para la Salud ${ }^{46}$. Una vez obtenidos los permisos de las instituciones correspondientes se capacitaron a los docentes (aplicadores) sobre el método y estrategia específica para una recolección de información homogénea. A través del consentimiento informado, cada participante recibió una explicación clara y completa del objetivo de la investigación, descripción de los procedimientos, riesgos y beneficios; se aseguró la confidencialidad y anonimato, con absoluta libertad de retirarse en cualquier momento si así lo deseaban. En todo momento se les indicó cómo moverse para evitar cualquier incidente; la aplicación de los instrumentos eventualmente podía desencadenar algunas situaciones de tristeza o incomodar al participante, por lo que, en estos casos, se suspendían temporalmente 
las preguntas y se escuchaba atentamente a la persona, en espera de que se sintiera mejor y se le preguntó si deseaba continuar en el estudio.

El procesamiento de los datos fue a través del paquete estadístico para las Ciencias Sociales (SPSS por sus siglas en inglés), versión 20 para Windows. Se utilizó estadística descriptiva: para las variables categóricas se determinaron frecuencias y porcentajes y para las variables continuas obtuvieron medidas de tendencia central y de dispersión. También se usó estadística inferencial no paramétrica, debido a que algunas variables no reportaron normalidad en la distribución de los datos (coeficiente de correlación de Spearman y prueba U de Mann Whitney); se empleó la prueba de KolmogorovSmirnov con corrección de Lilliefors. Para determinar la influencia de variables clínicas, demográficas y psicosociales sobre el autocuidado, se ajustó un modelo de regresión lineal múltiple con técnica Backward.

\section{RESULTADOS}

La muestra estuvo conformada por 96 participantes, con edad promedio de 45.6 años ( $\mathrm{DE}=9.7$ ); la escolaridad promedio equivale al nivel de secundaria. La mayoría están unidos, trabajan como empleados, presentaron problemas de sobrepeso u obesidad, no habían recibido educación en diabetes; casi la mitad del grupo acostumbra consumir alcohol (Tabla 1).

Clínicamente se caracterizaron por tener 5.5 años de diagnóstico, tres comorbilidades y generalmente reciben tratamiento oral. Se crearon dos categorías de acuerdo con la glucosa preprandial: con y sin control glucémico, predominando los pacientes sin control $(n=58)$; se encontró que los pacientes con control glucémico tuvieron menores cifras de circunferencia de cintura ( $p<.05)$, el resto de las variables fueron similares en ambas categorías (Tabla 2).

Tabla 1. Perfil sociodemográfico, clínico y de salud de la muestra

\begin{tabular}{|c|c|c|c|}
\hline Variables & Categoría & $M(D E)$ & $f(\%)$ \\
\hline Edad & & $45.6(9.7)$ & \\
\hline Escolaridad & & $8.9(3.7)$ & \\
\hline Estado civil & $\begin{array}{l}\text { Soltero/Divorciado/Viudo } \\
\text { Casado }\end{array}$ & & $\begin{array}{l}35(36.5) \\
61(63.5)\end{array}$ \\
\hline Ocupación & $\begin{array}{l}\text { Empleado } \\
\text { Negocio propio } \\
\text { Desempleado }\end{array}$ & & $\begin{array}{l}60(62.5) \\
13(13.5) \\
23(24.0)\end{array}$ \\
\hline IMC & $\begin{array}{l}\text { Normo-peso } \\
\text { Sobrepeso } \\
\text { Obesidad }\end{array}$ & & $\begin{array}{l}22(22.9) \\
40(41.7) \\
34(35.4)\end{array}$ \\
\hline Cintura & $\begin{array}{l}\text { Control } \\
\text { Riesgo de salud }\end{array}$ & & $\begin{array}{l}46(47.9) \\
50(52.1)\end{array}$ \\
\hline Educación en diabetes & $\begin{array}{l}\text { Si } \\
\text { No }\end{array}$ & & $\begin{array}{l}31(32.3) \\
65(67.7)\end{array}$ \\
\hline Tipo de tratamiento & $\begin{array}{l}\text { Oral } \\
\text { Insulina } \\
\text { Oral e insulina }\end{array}$ & & $\begin{array}{c}76(79.2) \\
16(16.7) \\
4(4.2) \\
\end{array}$ \\
\hline Monitoreo de glucosa & $\begin{array}{l}\mathrm{Si} \\
\mathrm{No}\end{array}$ & & $\begin{array}{l}57(59.4) \\
39(40.6)\end{array}$ \\
\hline Glicemia preprandial & & $160.3(58.3)$ & \\
\hline Glicemia postprandial & & $218.5(76.9)$ & \\
\hline Consumo de alcohol & $\begin{array}{l}\text { Si } \\
\text { No }\end{array}$ & & $\begin{array}{l}47(49.0) \\
49(51.0)\end{array}$ \\
\hline Consumo de tabaco & $\begin{array}{l}\mathrm{Si} \\
\text { No }\end{array}$ & & $\begin{array}{l}22(22.9) \\
74(77.1) \\
\end{array}$ \\
\hline
\end{tabular}

$M=$ Media, $\mathrm{DE}=$ Desviación Estándar 


\section{Análisis de correlación}

Como se muestra en la Tabla 3, las variables psicosociales depresión y angustia por diabetes se correlacionan positivamente y éstas con el número de comorbilidades. La autoeficacia se correlacionó de forma negativa con la depresión, la angustia, las comorbilidades y las horas que permanece sentado/parado; mientras que el autocuidado lo fue de manera positiva con la autoeficacia y negativa con la angustia por diabetes.

\section{Efecto de las variables centrales}

Con el propósito de determinar la influencia de variables independientes de tipo demográfico (edad y escolaridad), clínicas (años de evolución, horas sentado/parado, CC, IMC, consumo de alcohol y

Tabla 2. Análisis descriptivo de información relacionada a la diabetes

\begin{tabular}{lccccc}
\hline Variables (posible puntaje) & $\begin{array}{c}\text { General } \\
(\mathbf{n}=\mathbf{9 6})\end{array}$ & $\begin{array}{c}\text { Control } \\
\text { glucémico } \\
(\mathbf{n}=\mathbf{3 8})\end{array}$ & $\begin{array}{c}\text { No control } \\
\text { glucémico } \\
(\mathbf{n}=58)\end{array}$ & $\begin{array}{c}\text { U de Mann- } \\
\text { Whitney }\end{array}$ & Valor de $p$ \\
\hline IMC & $27.9 \pm 4.42$ & $27.8 \pm 3.94$ & $27.9 \pm 4.73$ & 1095.5 & .961 \\
\hline CC & $92.0 \pm 18.02$ & $90.0 \pm 16.14$ & $97.5 \pm 16.80$ & 824.0 & $.037^{*}$ \\
\hline TAS & $130.0 \pm 12.91$ & $130.0 \pm 15.14$ & $130.0 \pm 11.21$ & 1030.5 & .582 \\
\hline TAD & $80.0 \pm 8.54$ & $80.0 \pm 8.47$ & $80.0 \pm 8.63$ & 1023.0 & .528 \\
\hline Años de evolución & $5.5 \pm 5.51$ & $6.0 \pm 5.07$ & $5.0 \pm 5.82$ & 1043.0 & .656 \\
\hline Horas sentado/parado & $8.0 \pm 3.45$ & $8.0 \pm 3.92$ & $8.0 \pm 3.05$ & 877.0 & .087 \\
\hline Número de comorbilidades & $3.0 \pm 2.27$ & $3.0 \pm 2.60$ & $3.0 \pm 2.09$ & 808.0 & .788 \\
\hline Autocuidado (o-10o) & $36.0 \pm 16.90$ & $40.0 \pm 19.55$ & $36.0 \pm 15.05$ & 995.0 & .422 \\
\hline Autoeficacia (o-10o) & $64.5 \pm 23.29$ & $65.2 \pm 22.31$ & $63.8 \pm 24.09$ & 1063.0 & .770 \\
\hline Depresión (o-6o) & $9.0 \pm 11.85$ & $6.0 \pm 12.07$ & $11.5 \pm 11.72$ & 918.5 & .169 \\
\hline Angustia por diabetes $(>3)$ & $1.6 \pm 1.10$ & $1.5 \pm 0.98$ & $1.7 \pm 1.18$ & 992.5 & .410 \\
\hline
\end{tabular}

$C C=$ Circunferencia cintura, TAS = Tensión arterial sistólica, TAD = Tensión arterial diastólica,

$\mathrm{U}=$ Test estadístico de Mann-Whitney, ${ }^{*} \mathrm{p}<.05$

Tabla 3. Correlación de Spearman de las variables de estudio

\begin{tabular}{|c|c|c|c|c|c|c|c|c|c|c|c|c|c|c|}
\hline & 1 & 2 & 3 & 4 & 5 & 6 & 7 & 8 & 9 & 10 & 11 & 12 & 13 & 14 \\
\hline 1 & 1 & & & & & & & & & & & & & \\
\hline 2 & $.535^{* *}$ & 1 & & & & & & & & & & & & \\
\hline 3 & -.155 & $-.261^{*}$ & 1 & & & & & & & & & & & \\
\hline 4 & $-.202^{*}$ & $-.333^{* *}$ & $.597^{* *}$ & 1 & & & & & & & & & & \\
\hline 5 & -.059 & .010 & .138 & .050 & 1 & & & & & & & & & \\
\hline 6 & -.212 & $-.298^{* *}$ & $372^{* *}$ & $.376^{* *}$ & .171 & 1 & & & & & & & & \\
\hline 7 & $-.343^{* *}$ & $-.230^{*}$ & -.105 & .062 & -.126 & .045 & 1 & & & & & & & \\
\hline 8 & -.102 & -.012 & .085 & .111 & .141 & $.224^{*}$ & .184 & 1 & & & & & & \\
\hline 9 & -.134 & -.001 & .138 & .141 & -.097 & -.011 & -.020 & $.284^{* *}$ & 1 & & & & & \\
\hline 10 & .087 & .043 & -.056 & $.248^{*}$ & -.046 & .072 & .070 & -.054 & .118 & 1 & & & & \\
\hline 11 & -.060 & -.148 & .165 & $.293^{* *}$ & .124 & $.344^{* *}$ & .185 & .137 & -.115 & $.263^{* *}$ & 1 & & & \\
\hline 12 & .008 & -.197 & .137 & $.215^{*}$ & -.039 & .202 & $.265^{* *}$ & .089 & -.013 & .142 & $.450^{* *}$ & 1 & & \\
\hline 13 & .047 & -.004 & .118 & -.060 & $.318^{* *}$ & .092 & .064 & $.261^{*}$ & .140 & .019 & .136 & .155 & 1 & \\
\hline 14 & .193 & .088 & -.084 & -.189 & .042 & $-.242^{*}$ & -.081 & -.105 & -.194 & -.042 & -.013 & -.037 & -.189 & 1 \\
\hline
\end{tabular}

Nota: 1. Autocuidado, 2. Autoeficacia, 3. Depresión, 4. Angustia por diabetes, 5. Años de diagnóstico, 6. Comorbilidades, 7. Horas sentado/parado, 8. \# de hospitalizaciones por diabetes, 9. Glicemia, 10. Cintura, 11. Presión sistólica, 12. Presión diastólica, 13. Edad, 14. Escolaridad. ${ }^{*} \mathrm{p}<.05,{ }^{* *} \mathrm{p}<.01$ 
tabaco), psicológicas (depresión y angustia por diabetes) y la autoeficacia sobre el autocuidado como variable dependiente, se realizó un análisis de regresión lineal con técnica Backward. Las variables que resultaron significativas fueron: la autoeficacia, el consumo de alcohol, las horas de estar sentado/parado, el tiempo de diagnóstico y la edad al explicar el modelo en un 47.7\% del total de la varianza (Tabla 4).

Tabla 4. Análisis de regresión lineal para autocuidado en DMT2

\begin{tabular}{lccc}
\hline Variable & Modelo & \multicolumn{2}{c}{ IC 95\% } \\
\hline Edad & $\beta$ & Límite Inferior & Límite Superior \\
\hline Escolaridad & $.189^{*}$ & .022 & .307 \\
\hline Años de diagnóstico & .118 & -.082 & .617 \\
\hline IMC & $-.222^{* *}$ & -.590 & -.092 \\
\hline Horas sentado/parado & .141 & -.032 & .571 \\
\hline Consumo alcohol & $-.274^{* *}$ & -1.055 & -.286 \\
\hline Consumo tabaco & $-.297^{* *}$ & -7.904 & 1.125 \\
\hline Depresión & -.114 & -5.677 & .165 \\
\hline Angustia por diabetes & .011 & -.150 & .282 \\
\hline Autoeficacia & .052 & -1.322 & \\
\hline R2 & $.393^{* *}$ & .114 & \\
\hline F & .532 & & \\
\hline R2a & $9.65^{* *}$ & & \\
\hline${ }^{*}$ p<.05, ${ }^{* *}$ p <.o1. $\mathrm{R}^{2} \mathrm{a}=\mathrm{R}$ cuadrada ajustada. & & & \\
\hline
\end{tabular}

\section{DISCUSIÓN}

De acuerdo con los resultados, los participantes se caracterizaron por ser del sexo masculino, ser económicamente activos y tener nivel educativo de secundaria; indicaron nunca haber recibido educación sobre su enfermedad. La escolaridad se ha visto asociada con un bajo nivel de conocimientos sobre la diabetes ${ }^{47}$. Otros estudios mencionan que los hombres tienen poco conocimiento sobre su enfermedad y lo buscan por su propia cuenta ${ }^{24}$, por lo que el bajo nivel educativo en los hombres es una barrera para el manejo de la DMT2 ${ }^{11}$.

Los participantes tuvieron un bajo control glucémico y presencia de comorbilidades a pesar de los pocos años de diagnóstico. Aunado a esto, la mayoría de los entrevistados tenían sobrepeso/ obesidad y una circunferencia abdominal por encima de parámetros adecuados. Por lo anterior, la población se considera en riesgo cardiovascular y de padecer complicaciones crónicas ${ }^{4}$. El consumo de alcohol en los participantes también estuvo presente, probablemente asociado a su estilo de vida y afrontamiento a la enfermedad ${ }^{13,15}$.

Las variables autocuidado y autoeficacia mostraron bajos niveles de cumplimiento, incluso en quienes mantenían buen control glucémico. El autocuidado en hombres ha sido definido por De Keijzer ${ }^{49}$ como algo inexistente, en el que cuidarse es un rol meramente femenino. Lo anterior se puede explicar por la falta de aceptación de la enfermedad y la sobrestimación de ésta ${ }^{15}$. Las imágenes de la masculinidad como fortaleza, autoridad, responsabilidad y liderazgo influyen en las conductas de salud; el hombre necesita mantener una imagen de autocontrol y fuerza frente a los demás, lo que lo limita a ejecutar acciones de autocuidado ya que podría ser visto como débil24,50. 
Los niveles de depresión y angustia por diabetes en general resultaron bajos, lo que puede tener relación con la construcción de la masculinidad en la población latina, la cual dicta que el hombre debe ser capaz de soportar el dolor y las dificultades sin demostrar debilidad o queja 24,11,50.

El análisis bivariado indicó que a mayor autoeficacia existen menores niveles de depresión y angustia ante la diabetes7, 20, 51, así como menos comorbilidades ${ }^{7}$ y menos tiempo de permanecer sentado/parado. Estos hallazgos se respaldan en lo citado por autores que señalan a la autoeficacia como un factor importante que determina el buen cuidado de la diabetes ${ }^{21} \mathrm{e}$ influye indirectamente en el control glucémico a través de conductas de automanejo. Se ha observado que las personas que tienen confianza en sus capacidades, tienen mayor probabilidad de ejecutar el cumplimiento de tareas ${ }^{7}$ para sobrellevar y hacer frente a la diabetes, así como a condiciones frecuentes como angustia o incluso depresión; en este estudio ambas condiciones se relacionan con la autoeficacia, a pesar de que la prevalencia en los hombres fue baja.

A medida que se incrementan las acciones de autocuidado en diabetes, disminuyen los niveles de angustia y el tiempo de mantenerse sentado/parado. Asimismo, condiciones como la depresión y la angustia mantuvieron una fuerte relación con la presencia de comorbilidades. Estos hallazgos sugieren que la DMT2 es una condición que causa angustia por la complejidad en el manejo, lo cual se relaciona con otros problemas psicológicos como la depresión ${ }^{52}$; ambas contribuyen en el descontrol glucémico, lo que favorece la presencia de comorbilidades.

De acuerdo con los predictores del autocuidado, la autoeficacia fue la variable más representativa, dato similar a otros estudios7,24,53. Las acciones de autocuidado son aprendidas a través del tiempo y, para realizarlas, las personas deben desarrollar habilidades especializadas para cubrir las demandas específicas de acuerdo con su condición ${ }^{54}$. La autoeficacia y las conductas de autocuidado son parte esencial en el manejo de la cronicidad, por lo que es necesario incrementar el desarrollo de dichas habilidades en el cuidado de la DMT29.

Otro factor importante que influyó en el autocuidado, de manera negativa, fue el consumo de alcohol; estos datos han sido reportados en otras investigaciones, siendo mayor en hombres que en mujeres. El consumo de alcohol es parte de un estilo de vida no saludable, contrario a lo recomendado en el cuidado de la DMT2 ${ }^{13,15}$. Este consumo es más frecuente cuando los hombres no aceptan su enfermedad, de manera que difícilmente seguirán las recomendaciones médicas sin cambio en sus actividades diarias. En los hombres, el consumo de alcohol es parte de su identidad masculina y normas sociales; esta conducta se realiza hasta los últimos años de vida, bajo las condiciones y complicaciones que se encuentre el paciente.

El tiempo que los hombres permanecen sentados/parados mostró de forma inversa las acciones de autocuidado. Esto podría deberse a que el estilo de vida en el hombre o el tipo de trabajo representa una barrera en el autocuidado ${ }^{50}$. En este estudio los participantes se desempeñaban en largas jornadas, situación que no ayudaba a estructurar su vida de forma compatible con las acciones para el cuidado de su salud.

Los años de diagnóstico influyeron de forma inversa en el autocuidado. Esto difiere con otro estudio55, en el que el bajo control glucémico en personas se asocia con quienes tienen menos de seis años con la enfermedad. Lo anterior indica que la evolución de la diabetes es una condición que puede limitar el autocuidado y asociarse al descontrol glucémico. Por último, la edad fue un factor predictor del autocuidado, lo cual se ha reportado en otros estudios, al indicar que las personas con menor edad tienen peor control glucémico y prácticas de autocuidado deficientes en comparación con personas de mayor edad ${ }^{56,57}$. Estos resultados sugieren que los hombres jóvenes con DMT2 le dan 
poca importancia a su cuidado y que la experiencia adquirida del individuo sobre el manejo de su enfermedad será a medida que pasa el tiempo.

\section{CONCLUSIONES}

En relación con el objetivo del estudio, los resultados del modelo ofrecen mayor evidencia sobre los factores que influyen en el autocuidado del hombre: desde variables sociodemográficas como la edad; clínicas como los años de diagnóstico; conducta de riesgo como el consumo de alcohol y el trabajo en relación con las horas que permanece sentado o parado y principalmente la autoeficacia.

Los hallazgos del estudio concluyen que los hombres mantienen niveles bajos de depresión y angustia; sin embargo, se relacionan entre sí y también de forma directa con la autoeficacia, las acciones de autocuidado y las comorbilidades que presentan los hombres con DMT2. Además, sobresale la prevalencia del no control glucémico, la falta de educación en diabetes y la presencia de sobrepeso y obesidad.

Estos resultados sugieren la importancia del manejo de la depresión y angustia, por ser condiciones que con frecuencia no son expresadas por los hombres al considerarse una muestra de debilidad y, por lo tanto, no se diagnostican y tratan a tiempo. Lo anterior implica que los hombres no se acerquen a las instituciones de salud, lo que afecta el cumplimiento de las acciones de autocuidado.

El personal de enfermería debe entender los factores que influyen en el hombre para manejar su enfermedad, y realizar intervenciones que incrementen tanto la autoeficacia como las acciones de autocuidado para mejorar su control glucémico, y así retrasar las complicaciones. Futuras investigaciones deben dirigir sus esfuerzos en la planeación de intervenciones efectivas para los hombres, además de continuar indagando en los factores que influyen en el autocuidado y la autoeficacia mediante enfoques mixtos debido a la naturaleza del fenómeno.

\section{RESPONSABILIDADES ÉTICAS}

Protección de personas y animales. Los autores declaran que en este estudio no se realizaron experimentos en seres humanos y animales.

Confidencialidad. Los autores declaran que en el estudio no aparecen datos que permitan la identificación de los pacientes.

Derecho a la privacidad y consentimiento informado. Los autores declaran que han obtenido el consentimiento informado de los pacientes del estudio.

Conflicto de interés. Los autores declaran no tener conflicto de interés.

Financiamiento. Ninguno.

\section{REFERENCIAS}

1. IDF. Diabetes Atlas. Chapter 3 The global picture. $8^{\text {th }}$ edition United Kingdom: IDF; 2017: 40-65. https://bit.ly/2uIzTag

2. Instituto Nacional de Salud Pública. Encuesta Nacional de Salud y Nutrición de Medio Camino 2016 (ENSANUT MC 2016). México: SSA-INSP; 2016: 46-54. https://bit.ly/2HnwaSk

3. Instituto Nacional de Estadística y Geografía (INEGI). Características de defunciones registradas en México durante el 2017. México: INEGI; 2017. https://bit.ly/31OQcys

4. American Diabetes Association. Lifestyle Management: Standards of Medical Care in Diabetes. Diabetes Care. 2018; 41(1): 38-50. https://doi.org/10.2337/dc18-Soo4 
5. Instituto Mexicano del Seguro Social (IMSS). Diagnóstico y tratamiento farmacológico de la diabetes mellitus tipo 2 en el Primer Nivel de Atención. (Guía de Práctica Clínica). México: IMSS; 2018. https://bit.ly/2w7tUMr

6. Simplício-Oliveira P, Lopes-Costa MM, Lopes-Ferreira JD, Jácome-Lima CL. Autocuidado en diabetes mellitus: estudio bibliométrico. Enf. Global. 2017; 16(1): 634-52.

https://doi.org/10.6018/eglobal.16.1.249911

7. Lin K, Park Ch, Li M, Wang X, Li X, Li W, et al. Effects of depression, diabetes distress, diabetes selfefficacy, and diabetes self-management on glycemic control among Chinese population with type 2 diabetes mellitus. Diabetes Res Clin Pract. 2017; 131(38): 179-86.

http://dx.doi.org/10.1016/j.diabres.2017.03.013

8. Kav S, Yilmaz AA, Bulut Y, Dogan N. Self-efficacy, depression and self-care activities of people with type 2 diabetes in Turkey. Collegian. 2017; 24(1):27-35. https://doi.org/10.1016/j.colegn.2015.09.005

9. D'Souza MS, Karkada SN, Parahoo K, Venkatesaperumal R, Achora S, Cayaban AR. Self-efficacy and self-care behaviours among adults with type 2 diabetes. Appl Nurs Res. 2017; 36: 25-32.

http://dx.doi.org/10.1016/j.apnr.2017.05.004

10. Shrivastava S, Shrivastava P, Ramasamy J. Role of self-care in management of diabetes mellitus. J Diabetes Metab Disord. 2013; 12(1): 14. https://doi.org/10.1186/2251-6581-12-14

11. Mendoza-Catalán G, Juárez-Medina L, Pimentel-Jaimes JA, Higuera Sainz JL, Gutiérrez-Valverde JM. Barreras socioculturales en el manejo de la diabetes en hombres de origen mexicano: revisión sistemática. Nure Inv. 2017; 14(91): 1-10. https://bit.ly/3bAGNii

12. Garzón-Segura AM. Masculinidad y feminidad hegemónicas y sus consecuencias en la salud de hombres y mujeres. Al sur de todo. 2015; 10: 1-12. https://doi.org/10.13140/RG.2.1.2401.7689

13. Cruz-Bello P, Vizcarra-Bordi I, Kaufer-Horwits M, Benítez-Arciniega AD, Misra R, Valdés-Ramos R. Género y autocuidado de la diabetes mellitus tipo 2 en el Estado de México. Pap Poblac. 2014; 20(80): 119-44. https://bit.ly/37vybXi

14. Rossaneis MA, Haddad MC, Mathias TA, Marcon SS. Diferencias entre mujeres y hombres diabéticos en el autocuidado de los pies y estilo de vida. Rev. Latino-Am. Enfermagem. 2016; 24: e2761. http://dx.doi.org/10.1590/1518-8345.1203.2761

15. Mendoza-Catalán G, Chaparro-Díaz L, Gallegos-Cabriales E, Carreno-Moreno S. Automanejo en diabetes mellitus tipo 2 desde un enfoque de género: revisión integrativa. Enferm. univ. 2018; 15(1): 9o-102. http://dx.org.doi/10.22201/eneo.23958421e.2018.1.63276

16. Mendoza-Catalán G, Gallegos-Cabriales E, Gutiérrez-Valverde JM. Autocuidado del hombre con diabetes mellitus tipo 2: impacto del machismo. Enf global. 2018; 51:1-13.

http://dx.doi.org/10.6018/eglobal.17.3.287231

17. Bandura A. Self- efficacy mechanism in human agency. American Psychologist. 1982; 37(2): 122-147. https://bit.ly/2Sn4BPh

18. Amer FA, Mohamed MS, Elbur AI, Abdelaziz SI, Elrayah ZA. Influence of self-efficacy management on adherence to self-care activities and treatment outcome among diabetes mellitus type 2 Sudanese patients. Pharm Pract (Granada). 2018;16(4):1274. https://doi.org/10.18549/PharmPract.2018.04.1274

19. Adam J, Folds L. Depression, self-efficacy, and adherence in patients with type 2 diabetes. J Nurse Pract 2014; 10(9): 646-52. https://doi.org/10.1016/j.nurpra.2014.07.033

20. Brown SA, García AA, Brown A, Becker BJ, Conn VS, Ramírez G, et al. Biobehavioral determinants of glycemic control in type 2 diabetes: A systematic review and meta-analysis. Patient Educ Couns. 2016; 99(10): 1558-67. http://dx.doi.org/10.1016/j.pec.2016.03.020 
21. Devarajooh C, Chinna K. Depression, distress and self-efficacy: The impact on diabetes self-care practices. PLoS One. 2017; 12(3): 116. https://doi.org/10.1371/journal.pone.0175096

22. Walker RJ, Smalls BL, Hernandez-Tejada MA, Campbell JA, Egede LE. Effect of diabetes self-efficacy on glycemic control, medication adherence, self-care behaviors, and quality of life in a predominantly low-income, minority population. Ethn Dis. 2014; 24(3): 349-55.

23. González-Cantero J, González A, Vázquez CJ, Galán CS. Autoeficacia y adherencia terapéutica en personas con diabetes mellitus tipo 2. Rev. investig psicol. 2015; 18(1): 47-61.

https://doi.org/10.15381/rinvp.v18i1.11772

24. Guerrero-Alcedo JM, Parra-Soteldo LR, Mendoza-Oropeza JC. Autoeficacia y calidad de vida en pacientes con diabetes mellitus tipo 2 sometidos a hemodiálisis. Rev Cubana Salud Pública. 2016; 42(2): 193-203. https://bit.ly/38s3670

25. Perrin NE, Davies MJ, Robertson N, Snoek FJ, Khunt K. The prevalence of diabetes-specific emotional distress in people with type 2 diabetes: a systematic review and meta-analysis. Diabet. Med. 2017; 34(11): 1508-20. https://doi.10.1111/dme.13448

26. Antúnez $M$, Bettiol AA. Depresión en pacientes con diabetes tipo 2 que acuden a una consulta externa de medicina interna. Acta Med Colomb. 2016; 41(2): 102-10. https://bit.ly/2wg2KTQ

27. Chew BH, Vos R, Mohd-Sidik S, Rutten GE. Diabetes-related distress, depression and distressdepression among adults with type 2 diabetes mellitus in Malaysia. PloS One. 2016; 11(3): e0152095. https://doi.org/10.1371/journal.pone.0152095

28. Seidler ZE, Dawes AJ, Rice SM, Oliffe JL, Dhillon HM. The role of masculinity in men's help-seeking for depression: A systematic review. Clin Psychol Rev. 2016; 49: 106-18.

https://doi.org/10.1016/j.cpr.2016.09.002

29. Dieter T, Lauerer J. Depression or Diabetes Distress? Perspect Psychiatric Care. 2018; 54(1): 84-7. https://doi.org/10.1111/ppc.12203

30. Polonsky W, Fisher L, Earles J, Dudl, R, Lees J, Mullan J, et al. Assessing psychosocial stress in diabetes: Development of the Diabetes Distress Scale. Diabetes Care. 2005; 28(3): 626-31. https://doi.org/10.2337/diacare.28.3.626

31. Sadiq R, Batool A. Relationship of diabetes related distress with psychological distress with psychological distress in type 2 diabetic patients. J Postgrad Med Inst. 2017; 31(4): 405-9. https://bit.ly/2tZjYUI

32. Zhou H, Zhu J, Liu L, Li F, Fish AF, Chen T, et al. Diabetes-related distress and its associated factors among patient with type 2 diabetes mellitus in China. Psychiatry Res. 2017; 252: 45-50. https://doi.org/10.1016/j.psychres.2017.02.049

33. Kumar N, Unnikrishnan B, Thapar R, Mithra P, Kulkarni V, Holla R, et al. Distress and Its effect on adherence to antidiabetic medications Among type 2 diabetes patients in Coastal South India. J Nat Sci Biol Med. 2017; 8(2): 216-20. https://doi.org/10.4103/0976-9668.210008

34. Martinez-Vega IP, Doubova SV, Pérez-Cuevas R. Distress and its association with self-care in people with type 2 diabetes. Salud Ment. 2017; 4O(2): 47-55. http://dx.doi.org/10.17711/sm.0185-3325.2017.007

35. Jannoo Z, Wah YB, Lazim AM, Hassali MA. Examining diabetes distress, medication adherence, diabetes self-care activities, diabetes-specific quality of life and health-related quality of life among type 2 diabetes mellitus patients. J Clin Tranl Endocrinol. 2017; 9: 48-54. https://doi.org/10.1016/j.jcte.2017.07.003

36. Secretaría de Salud. Norma Oficial Mexicana NOM-008-SSA2-2017, para el tratamiento integral del sobrepeso y la obesidad. México: SSA; 2017. https://bit.ly/2SGacPK 
37. Secretaría de Salud. Proyecto de Norma Oficial Mexicana PROY-NOM-015-SSA2-2018, para la prevención, detección, diagnóstico, tratamiento y control de la diabetes mellitus. México: SSA; 2018. https://bit.ly/2P7ElXh

38. Secretaría de Salud. Norma Oficial Mexicana NOM-030-SSA2-2009, para la prevención, detección, diagnóstico, tratamiento y control de la hipertensión arterial Sistémica. México: SSA; 2009. https://bit.ly/2SpVOvW

39. Toobert DJ, Glasgow RE. Assessing diabetes self-management: the summary of diabetes self-care activities questionnaire. En: Bradley C. (Ed). Handbook of psychology and diabetes. A guide to psychological measurement in diabetes research and practice. Abingdon: Routledge; 1994: 351-75.

40. Gallegos-Cabriales E, Salazar-González B, Gutiérrez-Valverde JM. Teoría e investigación en autocuidado. Trabajo presentado en el primer Congreso Internacional de Modelos y teorías de enfermería: Transición de la teoría a la práctica. Colombia; 2011. https://bit.ly/31PMV1S

41. Loring KR, Ritter PL, González VM. Hispanic chronic disease self-management: a randomized communitybased outcome trial. Nurs Res. 2003; 52(6):361-9. https:/doi.org/10.1097/00006199-200311000-00003

42. Radloff LS. The CES-D Scale: a self-report depression scale for research in the general population. Appl Psychol Meas. 1977; 1(3): 385-401. https://doi.org/10.1177/014662167700100306

43. Salgado DN, Maldonado M. Características psicométricas de la escala de depresión del Centro de Estudios Epidemiológicos e mujeres mexicanas adultas de áreas rurales. Salud Pública Mex.1994; 36(2): 200-19. https://bit.ly/2uJDwNc

44. Bojorquez CL, Salgado DN. Características psicométricas de la escala Center for Epidemiological Studies-depression (CES-D), versiones de 20 y 10 reactivos, en mujeres de una zona rural mexicana. Salud Ment. 2009; 32(4): 299-307. https://bit.ly/2USioPo

45. SSA. Reglamento de la Ley General de Salud en Materia de Investigación para la Salud. México: DOF 02-04-2014; 1997. https://bit.ly/2uJ2pIP

46. Abdullah A, Liew SM, Salim H, Ng CJ, Chinna K. Prevalence of limited health literacy among patients with type 2 diabetes mellitus: A systematic review. PLoS ONE. 2019; 14(5): e0216402. https://doi.org/10.1371/journal.pone.0216402

47. Trujillo-Hernández B, Trujillo-Magallón E, Trujillo-Magallón M, Brizuela-Araujo CA, García-Medina MA, González-Jiménez MA, et al. Frecuencia del síndrome metabólico y factores de riesgo en adultos con y sin diabetes mellitus e hipertensión arterial. Rev. Salud Pública. 2017; 19(5): 609-16. https://doi.org/10.15446/rsap.V19n5.5696o

48. De Keijzer B. Hasta donde el cuerpo aguante: género, cuerpo y salud masculina. La salud como derecho ciudadano: perspectivas y propuestas desde América Latina. Lima, Perú: Foro Internacional en Ciencias Sociales y Salud; 2003: 137-52. https://bit.ly/2OVDkRY

49. Hawkins J, Watkins DC, Kieffer E, Spencer M, Piatt G, Nicklett EJ, et al. An exploratory study of the impact of gender on health behavior among african american and latino men with type 2 diabetes. Am J Mens Health. 2017; 11(2): 344-56. https://doi:10.1177/1557988316681125

50. Gharaibeh B, Gajewski BJ, Al-smadi A, Boyle DK. The relationships among depression, self-care agency, self-efficacy and diabetes self-care management. J Res Nurs. 2016; 21(2): 123-4. https://doi.10.1177/1744987115621782

51. Twist K, Stahl D, Amiel SA, Thomas S, Winkley K, Ismail K. Comparison of depressive symptoms in type 2 diabetes using a two-stage survey design. Psychosom Med. 2013; 75(8): 791-7.

http://doi:10.1097/PSY.obo13e3182a2b108 
52. Wichit N, Mnatzaganian G, Courtney M, Schulz P, Johnson M. Randomized controlled trial of a family-oriented self-management program to improve self-efficacy, glycemic control and quality of life among Thai individuals with Type 2 diabetes. Diabetes Res Clin Pract. 2017; 123: 37-48. http://doi.org/10.1016/j.diabres.2016.11.013

53. Orem DE. Nursing concepts of practice. $6^{\mathrm{a}}$ ed. St. Louis: Mosby; 2001.

54. Guo X, Weng J, Lu J, Yang W, Jia W, Zou D, et al. Determinants of poor glycemic control in Chinese men with type 2 diabetes: a cross-sectional survey of 15,427 men in 77 tertiary hospitals in China. Int J Diabetes Dev Ctries. 2015; 35(4): 488-92. https://doi10.1007/s13410-015-0322-3

55. Nanayakkara N, Ranasinha S, Gadowski AM, Davis WA, Flack JR, Wischer N, et al. Age-related differences in glycaemic control, cardiovascular disease risk factors and treatment in patients with type 2 diabetes: a cross-sectional study from the Australian National Diabetes Audit. BMJ Open. 2018; (8): eo20677. https://doi.org/10.1136/bjmopen-2017-020677

56. Casagrande S, Cowie CC, Fradkin JE. Intensive glycemic control in younger and older U.S. adults with type 2 diabetes. J Diabetes Complications. 2017; 31(8). https://doi.org/10.1016/j.jdiacomp.2017.05.006 